\title{
Urteilsbegründung zur Prüfpflicht von Bewertungsportalen
}

Der Bundesgerichtshof veröffentlichte kürzlich seine schriftliche Urteilsbegründung zum am 1. März 2016 gesprochenen Urteil und präzisierte die Prüfpflicht von (Arzt-) Bewertungsportalen (VIZR 34/15). Darin heißt es, dass Bestätigungen, Belege oder andere Dokumente von Patienten angefordert und an den bewerteten Arzt weitergeleitet werden müssen, wenn dieser bezweifelt, dass es sich um einen Patienten des Arzts handelt. Weiter betonten die Bundesrichter erneut die Bedeutung der Anonymität der Verfasser von Bewertungen, sodass die Weiterleitung der Patientenbestätigung nur soweit zu erfolgen hat, als dies ohne Verletzung der Anonymität ( $§ 12$ Telemediengesetz) erfolgen kann. Zudem bestätigten sie, dass es sich bei den Noten, die Patienten ihren Ärzten auf jameda in verschiedenen Kategorien geben, um Meinungsäußerungen und keine Tatsachenbehauptungen handelt. Auch bekräftigten sie, dass Portalbetreiber wie jameda eine „gesellschaftlich erwünschte Funktion" erfüllen und dass die konkreten Überprüfungspflichten hinsichtlich angegriffener Tatsachenbehauptungen jeweils im Einzelfall abzuwägen seien. Weitere Informationen darüber, wie jameda die Authentizität von Arztbewertungen sicherstellt, finden Sie unter: http://www. jameda.de/qualitaetssicherung/echte-bewertungen/

Nach einer Pressemitteilung der Jameda GmbH, München gesundheit@jameda.de 